\title{
Strictly Approximating Attributes of DNA Lesion Repair in Terms of Inherent DNA Responses to Mutability and Malignant Transformation
}

\author{
Lawrence MA* \\ Department of Pathology, University of Malta Medical School, Mater Dei Hospital, \\ Malta Europe
}

*Corresponding author: Lawrence M Agius, Department of Pathology, University of Malta Medical School, Mater Dei Hospital, 27 "Ballarat" Guzeppe Caruana Street, TalVirtu, Rabat Malta, Malta Europe, Tel: 356-21451752; E-mail: lawrence.agius@um.edu.mt

\begin{abstract}
Selective susceptibilities of the cellular genome for permissive emergence of mutability hot-spots are governed by specific patterns of approximate repair of lesions in terms of response to DNA damage. Given the pattern recognition of mutability as an integral approximation there is central cooperative susceptibility within such pattern responses that specify the attributes of genomic instability as contexts for approximate DNA repair. Malignant transformation is hence a para-physiologic consequence for permissive evolution of the malignant transformation of cells and for the creation of a milieu for permissive accumulation of mutability susceptibilities towards malignancy.
\end{abstract}

Keywords: Neuroblastoma; Nicotinamide; Dinucleotid; Phosphodiester; Isoform

Abbreviations: MYCN: V-Myc Avian Myelocytomatosis Viral Oncogene Neuroblastoma Derived Homolog; RNA: Ribonucleic acid; PARP: Poly (ADP-ribose) polymerases; TXNIP: Thioredoxininteracting Protein

\section{Introduction}

Substantial constitutional mechanisms employ a variety of mechanisms to protect and repair DNA damage of cells within the further dimensional extensions of malignant cells as these progress within systems of perforce proliferation and spread. Within the context of neuroblastoma, p53 and MYCN are mutually transcriptionally regulated and they together control the catalytic activity of RNA polymerases [1]. Prototypical of such events is translesional synthesis polymerase functionality and dysfunctionality in exposing liability towards neoplastic transformation. Pol eta bypasses lesions that block replicative polymerases and p53 favours such bypass by pol eta [2].

Dimensional cooperative action refers to the significant mutagenesis that promotes further genomic instability. Resveratrol improves efficacy of sorafenib-induced 


\section{Medical Journal of Clinical Trials \& Case Studies}

apoptosis in human breast cancer MCF7 cells by modulating reactive oxygen species, cell cycle inhibition, caspase 3 and PARP cleavage [3].

Breast cancers occurring in BRCA1 mutation patients are largely triple-negative and implicate homologous recombination deficiency, sensitising them to inhibition of a second DNA damage repair pathway as by PARP inhibitors [4].

The inherent tendencies of translesional synthesis polymerase action promote the incorporation of mutational base substitutes as further promotional increment towards diversity as seen in immunoglobulin synthesis and also towards a mutability that is central both for carcinogenesis and also for chemo- and radiotherapeutic resistance. Poly (ADP-ribose) polymerases (PARPs) catalyse PARylation by conjugating ADP-ribose residues repeatedly on amino acid residues using nicotinamide adenine dinucleotide as a substrate [5]. PARPylation is a widespread post-translation modification at DNA lesions and modulates chromatin reorganization, DNA damage response, transcriptional regulation, apoptosis and mitosis [6].

\section{Interplay of Cooperations}

DNA polymerases are involved in the replication, repair and recombination of genetic elements and also require two divalent metal ions for the phosphodiester bond formation [7].

The dynamics of replication proteins during lagging strand synthesis implicate the potential development of genomic instability and cancer; these involve the formation of small DNA fragments (Okazaki fragments) that lead to the synthesis of a continuous DNA strand [8]. The further cooperative interplay as determined by the bypass of DNA adducts as prototypically represented by alkylating chemotherapy and also after exposure to oxygen free radicals and ultraviolet radiation exposure.

Template DNA damage generally induces replication arrest and this can be released by translation DNA synthesis and template switching by homologous recombination; HERC2 and RNF8 favour TLS past basic sites and ultraviolet-lesions close to or at stalled replication forks [9].

It is relative to dysfunctionality in particular that carcinogenesis further evolves as constitutional and inherent susceptibility within the spectral exposure to both endogenous and exogenous agents that destabilize the genome. The PARP-1 enzyme is involved in the repair of ROS-induced DNA damage and blocking this repair with olaparib may permit accumulation of damage leading to massive apoptosis [10].

The processivity of individual family members of translesional polymerases is reflected in an often -limited number of nucleotides that are incorporated opposite normal or damaged DNA sequences.

The further promotional dimensions for cooperative activity of various translesional polymerases is a potentially highly effective system of an extensive series of targets in modulating chemotherapy and radiotherapy resistance inherent to evolutional steps in carcinogenesis. PARP inhibitors are primarily employed in malignancies with known defects in DNA repair genesis such as alterations in breast cancer, early onset $1 / 2$ (BRCA1/2) [11].

The further spectral exposure of mammalian cells to mutagenesis is substantial contribution to the initiation and further progression of multiple types of neoplasia as an important series of hotspots in carcinogenesis. PARP1 impacts DNA repair and apoptosis and PARP- 1 inhibitors are potent anticancer agents, including children with acute lymphoblastic leukemia [12]. Such stereotypical acquisition of DNA lesions is further confirmatory to the attributes of the central malignant transformational event itself. The counteracting effects of PARP synthesis on Exonuclease 1 recruitment and enzymatic activity may potentiate appropriate resection of DNA ends while preventing unscheduled or inappropriate processing of DNA breaks in cells [13]. The significant stereotypia is inherent to the emergence of tumors as response pathways to DNA damage, and indicates the universal susceptibility to malignant transformation in terms of individual agents within complex further promotional dimensions of mutability of bases and nucleotides of mammalian cells.

\section{Promotional Susceptibilities}

Cisplatin-resistant non-small cell lung cancer cells involve the overexpression and over-activation of PARP1 and the down regulation of pyridoxal kinase, relative to elevated apoptosis resistance [14]. Promotional accumulation of mutations is a system series of inherent proportional characterizations as significantly portrayed by translesional mutability and progressive carcinogenesis. It is the tendency to render the DNA more 


\section{Medical Journal of Clinical Trials \& Case Studies}

susceptible to mutability that identifies further evolutionary attributes of carcinogenesis that is also resistant to chemotherapeutic and radiotherapy.

A particularly intriguing aspect of tumorigenesis is the accumulative capacity of the cellular genome to further respond to DNA damage and also the characterization of particular suppressor genes or oncogenes to substantially support mutability of essentially susceptibility of DNA to further malignant transformation. Combined effects of beta-Lapachone and AP0866 on pancreatic cancer cell death via reactive oxygen species production and activated PARP1 [15] may prove significant.

Included as system pathways of metabolism of cellular DNA is the performance of damage response that dynamically incorporates the lesional identity as susceptibility hotspots in mutagenesis.

Cytidine Deaminase deficiency induces disequilibrium of the pyrimidine pool and inhibits PARP-1 function, promoting under-replication of DNA [16]. Bypass spectrum delineation is the overall characterization of adduct bypass by polymerases that may also be inherently capable of DNA extension in the added milieu of significant emergence of the malignant cell in transformation.

\section{Resistance Therapeutics}

Resistance therapeutics appears enhanced by translesional polymerase activity that is contextually referable to dynamic attributes of cellular and metabolic response to DNA damage in a manner that also arises as mutability inherent to polymerase-induced bypass of lesions or adducts. The diverse biological process that require PARPs and ADP-ribosylation in response to genotoxic stresses also includes regulation of chromatin structure, gene expression and RNA processing in biological fields that include cancer, inflammation and metabolism [17]. Developmental dimensions allow for an apparently inherent susceptibility to the mutational susceptibility patterns. In fact, the proportional response to DNA lesions is a compounded attribute for DNA attempts to repair mutational hotspots in its own right.

The subsequent cascade events thus allow permissive mutability as a characterization of the integrally single malignant transformation event as individual cells and as whole sub-sets of such cells. DNA polymerase beta is key to protection against oxidative DNA lesions via its function in base excision repair and the normal functionality of the dRP lyase is critical to maintained replication for integrity and helps prevent replication fork collapse to double strand breaks and cell transformation [18].

BRCA1/2 -mutant cells are hypersensitive to inactivation of poly (DNA-ribose) polymerase 1; this may not be limited to BRCA mutation carriers but include deficiencies in other components of the repair pathways [19]. Performance increments of susceptibility is hence the referential central pattern for dimensional carcinogenesis per se and allows for the characterization of translesional polymerases in terms of ongoing DNA lesion infliction on the one hand and of DNA response to damage to the cellular genome. Performance dynamics of repair of DNA damage is hence the central attribute to system susceptibility to an integral DNA damage that is rendered prototypically a systematic exposure for further mutability. Defects in the ubiquitin machinery potentially influence response of neoplastic cells to PARP inhibitors, via defective homologous recombination DNA repair [20].

\section{DNA Mutability}

The complex creativity for DNA mutability is a series of spectral susceptibilities that help define the nature of malignant transformation in terms of both qualitative attributes as also characterized specifically and also accumulative phenomena of mutational lesions. Stem cell differentiation studies have revealed paused genes that implicate the contemporary presence of both activator and repressor epigenetic markers (bivalent marking); TXNIP is an on co-suppressor gene whose level of expression is reduced in breast cancer and is a potential target for treatment [21].

The performance bypass of lesions is allied to primerstimulatory changes in extension of the DNA sequence. Genomic rearrangements are highly prevalent in prostate cancer and are compartmentalised into chromatin loop domains by the attachment to the nuclear matrix and are demonstrable in several recombination hot spots at the base of loops [22].

Approximation of dimensional DNA repair is constitutive identification of the malignant transformational event in its own right and within the inherent performance attributes of response to such DNA damage.

Mutability of DNA lesions is hence the distributional performance for further mutability within an approximate spectrum of DNA damage as borne out by many translesional polymerases and as also evidenced by 


\section{Medical Journal of Clinical Trials \& Case Studies}

susceptibilities of hotspot targets in evolving carcinogenesis. Exogenous p53 or TAp63 isoform can rescue the apoptotic program in response to treatment, and PARP inhibitors may operate in the choice between growth arrest and apoptosis through modulation of p53 family dependent signaling [23].

\section{Linkage Dynamics}

A linkage dynamics is hence strict characterization of the inherent attributes for DNA mutability in view of the series of changes in developmental emergence of a given neoplastic lesion. The degree for performance reactivity is significant attribute of most mammalian cells that permit the institution of particularly accumulative events in terms of ongoing genomic instability.

Pattern approximation of repair processes targeting DNA lesions is therefore an institutional series of inherent characterizations that include both qualitative and quantitative dimensions in mutability that in turn distribute hotspot susceptibilities to malignant transformation.

Promotion of translesional mutability as induced by many polymerases is hence the subset specification of the integral event in malignant transformation of cells and of groups of cells.

Damaged DNA hence is effectively an inducer of approximate repair in a strict contextual setting for performance extension from primer sites resulting from excisional repair of bases and nucleotides.

\section{Concluding Remarks}

A series of spectral mutabilities is susceptible to an apparently inherent tendency of damaged DNA for approximate repair of hotspot lesions within the mammalian DNA. The conceptual setting for further accumulative mutability is a specific modulated event that promotes performance susceptibility for further mutational change. Performance dynamics permit the emergence of multiple hotspot susceptibilities within further conformational mutability as well-illustrated by translesional synthesis polymerase action. Lesion repair is hence a mechanism for permissive DNA repair within the further setting of mutational targeting and incorporation.

\section{References}

1. Singhal SS, Singhal S, Singhal P, Singhal J, Home D, et al. (2017) Didymin: an orally active citrus flavonoid for targeting neuroblastoma. Oncotarget 8(17): 29428-29441.

2. Lerner LK, Francisco G, Soltys DT, Rocha CR, Quinet A, et al. (2017) Predominant role of DNA polymerase eta and p53-dependent translation synthesis in the survival of ultraviolet-irradiated human cells. Nucleic Acids Res 45(3):1270-1280.

3. Mondal A, Bennett LL (2016) Resveratrol enhances the efficacy of sorafenib mediated apoptosis in human breast cancer MCF7 cells through ROS, cell cycle inhibition, caspase 3 and PARP cleavage. Biomed Pharmac other 84: 1906-1914.

4. Heerma van Voss MR, Brilliant JD, Vesuna F, Bol GM, van der Wall E, et al. (2017) Combination treatment using DDX3 and PARP inhibitors induces synthetic lethality in BRCA1-proficient breast cancer. Med Oncol 34(3): 33.

5. Sasaki Y, Hozumi M, Fujimori H, Murakami Y, Koidzumi F, et al. (2016) PARG inhibitors and functional PARG inhibition models. Curr Protein Pept Sci 17(7): 641-653.

6. Wei H, Yu X (2016) Functions of PARylation in DNA Damage Repair pathways. Genomics Proteomics Bioinformatics 14(3):131-139.

7. Zhang Y, Baranovskly AG, Tahirov ET, Tahirov TH, Pavlov YI (2016) Divalent ions attenuate DNA synthesis by human DNA polymerase alpha by changing the structure of the template/primer or by perturbing the polymerase reaction. DNA Repair (Amst) 43: 24-33.

8. Deshmukh AL, Kumar C, Singh DK, Maurya P, Banerjee D (2016) "Dynamics of replication proteins during lagging strand synthesis: a crossroads for genomic instability and cancer". DNA Repair (Amst) 42:72-81.

9. Mohiuddin, Kobayashi S, Keka IS, Guilbaud G, Sale J, et al. (2016) The role of HERC2 and RNF8 ubiquitin E3 ligases in the promotion of translation DNA synthesis in the chicken DT40 cell line. DNA Repair (Amst) 40: 67-76.

10. Deben C, Lardon F, Wouters A, Op de Beeck K, Van den Bossche J, et al. (2016) APR-246 (PRIMA-1(MET)) 


\section{Medical Journal of Clinical Trials \& Case Studies}

strongly synergies with AZD2281 (olaparib) induced PARP inhibition to induce apoptosis in non-small cell lung cancer cell lines. Cancer Lett 375(2): 313-322.

11. Kivlin CM, Watson KL, Al Sannaa GA, Belousov R, Ingram DR, et al. (2016) Poly(ADP) ribose polymerase inhibition: a potential treatment of malignant peripheral nerve sheath tumor. Cancer Biol Ther 17(2): 129-138.

12. Kruk A, Oliepa T, Urasinski T, Grabarek J, Urasinska E (2015) PARP1 expression in CD34+ leukaemic cells in childhood acute lymphoblastic leukaemia: relation to response to initial therapy and other prognostic factors. Pol J Pathol 66(3):239-245.

13. Cheruiyot A, Paudyal SC, Kim IK, Sparks M, Ellenberger T, et al. (2015) Poly(ADP-ribose)-binding promotes Exo1 damage recruitment and suppresses its nuclease activities. DNA Repair (Amst) 35:106115 .

14. Michels J, Adam J, Goubar A, Obrist F, Demotte D, et al. (2015) Negative prognostic value of high levels of intracellular poly(ADP-ribose) in non-small cell lung cancer. Ann Oncol 26(12):2470-2477.

15. Breton CS, Aubry D, Ginet V, Puyal J, Heulot M, et al. (2015) Combinative effects of beta-Lapachone and AP0866 on pancreatic cancer cell death through reactive oxygen species production and PARP1 activation. Biochimie 116: 141-153.

16. Gamble $S$, Abuja A, Buhagiar-Labarchede G, OnclercqDelic R, Dairou J, et al. (2015) Pyrimidine pool disequilibrium induced by a cytidine deaminase deficiency inhibits PARP-1 activity, leading to the under-replication of DNA. PLoS Genet 11(7): e1005384.
17. Kraus WL (2015) PARPs and ADP-ribosylation: 50 years...and counting. Mol Cell 58(6): 902-910.

18. Rozacky J, Nemec AA, Sweasy JB, Kidane D (2015) Gastric cancer associated variant of DNA polymerase beta (Leu22Pro) promotes DNA replication associated double strand breaks. Oncotarget 6(27): 24474-24487.

19. Wesierska-Gadek J, Mauritz M, Mitulovic G, Cupo M (2015) Differential potential of pharmacological PARP inhibitors for inhibiting cell proliferation and inducing apoptosis in human breast cancer cells. J Cell Biochem 116(12): 2824-2839.

20. Frankum J, Moudry P, Brough R, Hodny Z, Ashworth A, et al. (2015) Complementary genetic screens identify the E3 ligase CBLC, as a modifier of PARP inhibitor sensitivity. Oncotarget 6(13): 10746-10758.

21. Baldan F, Mio C, Lavarone E, Di Loreto C, Puglisi F, et al. (2015) Epigenetic bivalent marking is permissive to the synergy of HDAC and PARP inhibitors on TXNIP expression in breast cancer cells. Oncol Rep 33(5): 2199-2206.

22. Barboro P, Ferrari N, Capaia M, Petretto A, Salvi S, et al. (2015) Expression of nuclear matrix proteins binding matrix attachment regions in prostate cancer. PARP-1: New player in tumor progression. Int J Cancer 137(7): 1574-1586.

23. Montariello D, Troiano A, Di Girolamo D, Beneke S, Calabro V, et al. (2015) Effect of poly(ADP-ribose) polymerase and DNA topoisomerase I inhibitors on the p53/p63-dependent survival of carcinoma cells. Biochem Pharmacol 94(3): 212-219. 